CORRECTION

\title{
Correction: Sleep disturbance in PTSD and other anxiety- related disorders: an updated review of clinical features, physiological characteristics, and psychological and neurobiological mechanisms
}

\author{
Anne Richards ${ }^{1,2}$, Jennifer C. Kanady ${ }^{1,2}$ and Thomas C. Neylan ${ }^{1,2}$ \\ Neuropsychopharmacology (2020) 45:240-241; https://doi.org/10.1038/s41386-019-0529-y
}

Correction to: Neuropsychopharmacology https://doi.org/10.1038/ s41386-019-0486-5, published online 23 August 2019.

Figure 3 has been replaced to properly label the amygdala and better depict the insula. The Figure 3 legend has also been amended.
Figure 5 has been replaced to situate the LC and the VLPO/IN consistently across sub-figures and more accurately with respect to their true brain anatomy.

This has now been corrected in both the PDF and HTML versions of the Article.
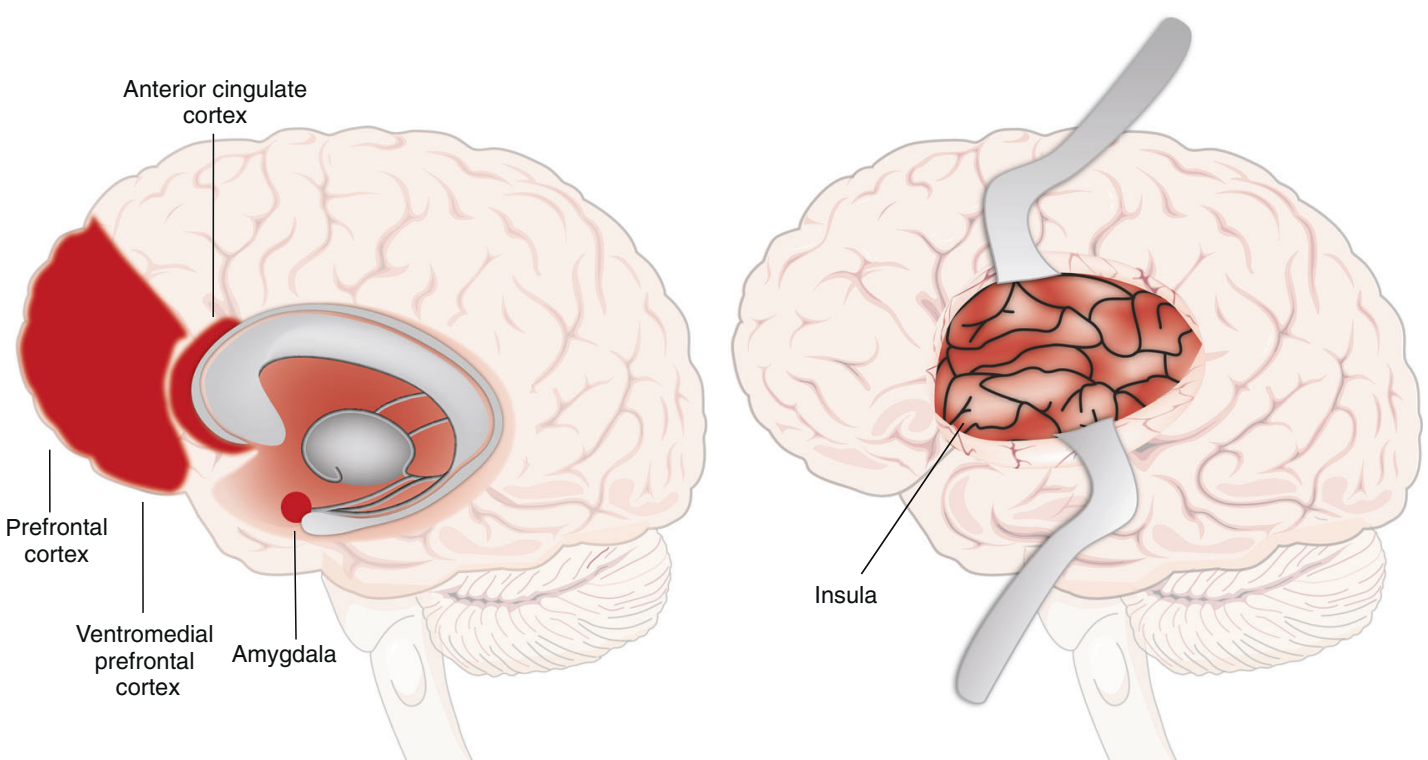

Fig. 3 Important brain regions involved in fear, threat, and anxiety expression and modulation. Neuroimaging research indicates that the amygdala and insula are involved in the expression of fear, threat and anxiety. The dorsal anterior cingulate cortex (dACC) is more involved in the processing and expression of anxiety and fear, whereas the rostral ACC ( $\mathrm{ACCC}$ ) is more involved in their modulation. The medial prefrontal cortex (mPFC) is involved in their modulation, especially the ventromedial PFC (vmPFC)

${ }^{1}$ The San Francisco VA Health Care System, San Francisco, CA, USA and ${ }^{2}$ The University of California, San Francisco, San Francisco, CA, USA Correspondence: Anne Richards (Anne.richards@ucsf.edu)

These authors contributed equally: Dr. Richards, Dr. Kanady

Published online: 7 October 2019 
a

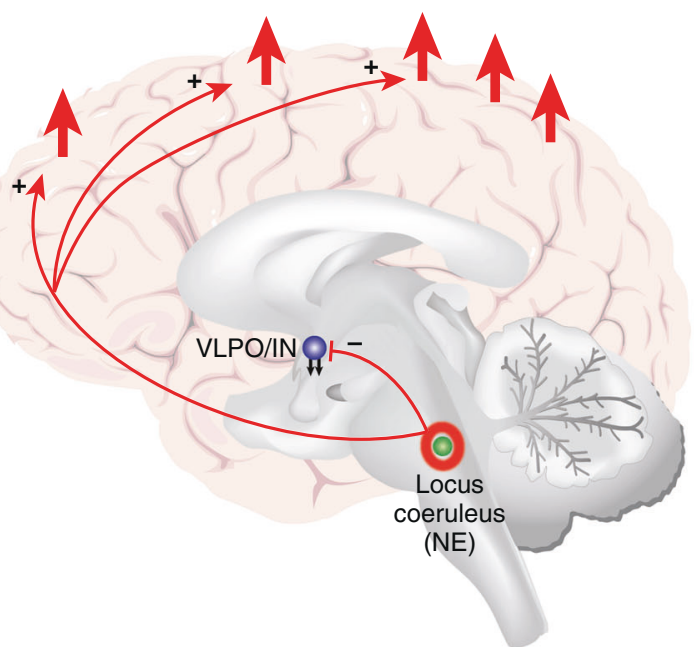

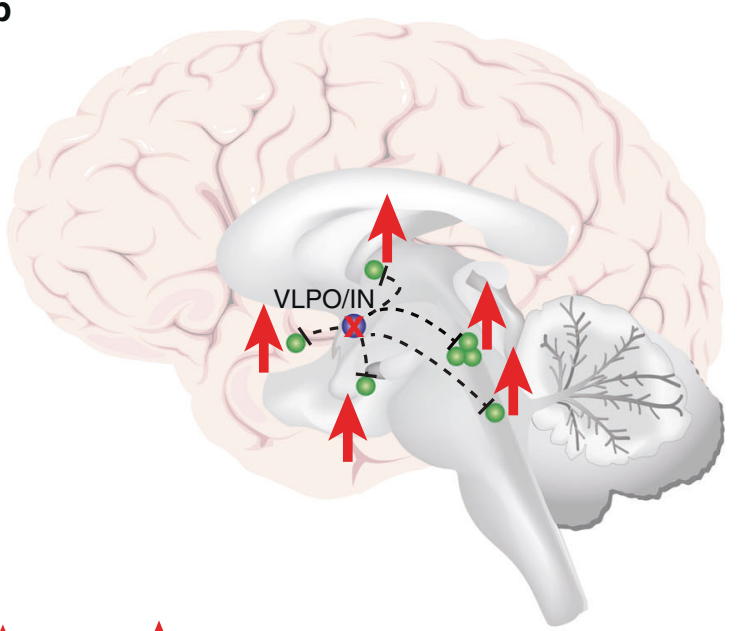

C

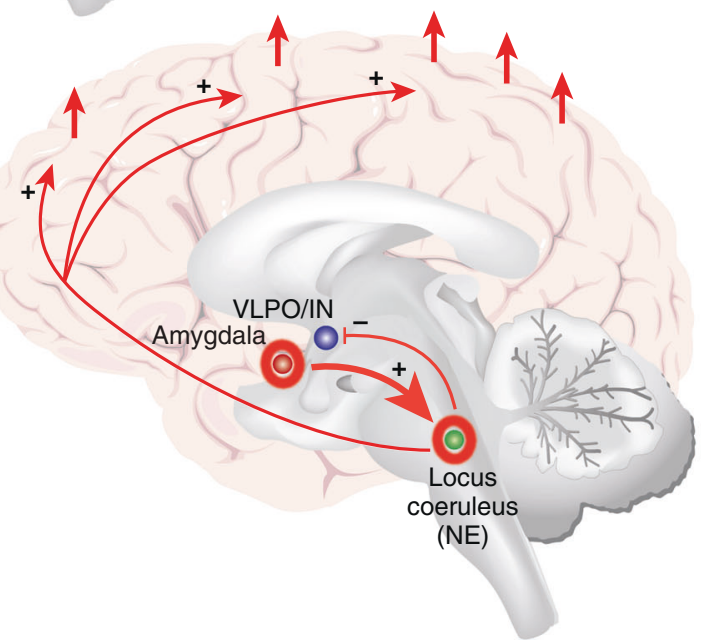

Fig. 5 Heuristic neurobiological framework for the sleep disturbance and anxiety disorder relationship. Abnormal activation in wake, NREMS, and/or anxiety regions may generate sleep disturbance in anxiety-related disorders and promote a bidirectional sleep-disturbance-anxiety relationship. a Sleep disturbance emerges from hyperactivation in intrinsic wake circuitry. For example, here, excess arousal in NE-generating LC (green with orange halo) results in inhibition of VLPO/Intermediate Nucleus (IN) (blue) and hyperactivation of cerebral cortex. b Sleep disturbance emerges from dysfuncton in central (NREM) sleep-promoting region. For example, here failure of VLPO GABA signaling (blue, with orange " $X$ ") disinhibits arousal centers (green). c Sleep disturbance emerges from hyperactivation of fear/threat/anxiety regions. For example, here amygdala hyperactivation (red, with orange halo) sends excitatory inputs to LC, which then inhibits VLPO-mediated sleep promotion and sends excitatory inputs to cerebral cortex, resulting in cortical hyperarousal, as in a. A common node in these examples is LC and NE signaling, but aberrant activity in other wake, NREMS, REMS, and anxiety regions may produce analogous effects 